\title{
Caractérisation Biochimique et Microbiologique de Soumbala de néré (Parkia biglobosa) et d'oseille de Guinée (Hibiscus sabdariffa) Produits au Niger
}

\author{
Roukaya Abdou Souley, \\ Halima Oumarou Diadié,
}

Laboratoire de Recherche en Hygiène, Sciences Alimentaires et Nutritionnelles (LRH-SAN), Faculté d'Agronomie, Université Abdou

Moumouni, Niamey, Niger

\section{Alio Sanda Abdelkader, Bakasso Yacoubou,}

Laboratoire de Gestion et Valorisation de la Biodiversité au Sahel GeVaBioS, Faculté des Sciences et Techniques, Département de Biologie,

Université Abdou Moumouni, Niamey, Niger

\section{Abdourahamane Balla,}

Laboratoire de Recherche en Hygiène, Sciences Alimentaires et Nutritionnelles (LRH-SAN), Faculté d'Agronomie, Université Abdou

Moumouni, Niamey, Niger

Doi:10.19044/esj.2020.v16n3p224～URL:http://dx.doi.org/10.19044/esj.2020.v16n3p224

\section{Résumé}

Le soumbala, condiment très consommé en milieu rural, demeure peu caractérisé au Niger. La présente étude vise à déterminer les caractéristiques physico-chimiques et microbiologiques des échantillons de soumbala de néré et d'oseille de guinée produits en milieu communautaire et au laboratoire. Pour ce faire, quatre échantillons de chacune des deux variantes de soumbala ont été analysés. Ainsi, la détermination de la composition physico-chimique des soumbala a été réalisée selon la méthode AOAC (1990). Le profil microbiologique a été évalué suivant les méthodes horizontales pour la détection des microorganismes dans les aliments. Les résultats ont montré que les teneurs moyennes en eau, protéines, lipides, glucides, énergie et minéraux ont varié en fonction de la typologie des soumbala et leur milieu de provenance. Par ailleurs, le profil microbien a montré la présence d'une microflore importante et variée, quel que soit la typologie des soumbala et leur milieu de provenance. Toutefois, les teneurs de ces microorganismes ont été en général à la limite des seuils d'acceptabilité des normes de références appliquées dans les cas de cette étude. Ceci indique le rôle indéniable que 
jouent les conditions environnementales sur la qualité des produits élaborés. Cette étude constitue une référence dans l'appréhension de la qualité hygiénique et nutritionnelle du soumbala du Niger.

Mots clés : Soumbala, Physico-Chimie, Microbiologie, Milieu De Production, Niger

\title{
Biochemical and Microbiological Characteristics of soumbala from Parkia biglobosa and Hibiscus sabdariffa produced in Niger
}

\author{
Roukaya Abdou Souley, \\ Halima Oumarou Diadié,
}

Laboratoire de Recherche en Hygiène, Sciences Alimentaires et Nutritionnelles (LRH-SAN), Faculté d'Agronomie, Université Abdou

Moumouni, Niamey, Niger

\section{Alio Sanda Abdelkader, Bakasso Yacoubou,}

Laboratoire de Gestion et Valorisation de la Biodiversité au Sahel GeVaBioS, Faculté des Sciences et Techniques, Département de Biologie,

Université Abdou Moumouni, Niamey, Niger

\section{Abdourahamane Balla,}

Laboratoire de Recherche en Hygiène, Sciences Alimentaires et Nutritionnelles (LRH-SAN), Faculté d'Agronomie, Université Abdou

Moumouni, Niamey, Niger

\begin{abstract}
Soumbala, a condiment widely consumed in rural areas, remains poorly characterized in Niger. The purpose of this study is to determine the physico-chemical and microbiological characteristics of nerve and guinea sorrel soumbala samples produced in the community and in the laboratory. To do this, four samples of each of the two variants of soumbala were analyzed. Thus, the determination of the physico-chemical composition of soumbala was carried out according to the AOAC method (1990). The microbiological profile was assessed using horizontal methods for the detection of microorganisms in food. The results showed that the average contents of water, proteins, lipids, carbohydrates, energy and minerals varied according to
\end{abstract}


the typology of soumbala and their environment of origin. In addition, the microbial profile showed the presence of a large and varied microflora, whatever the typology of soumbala and their environment of origin. However, the contents of these microorganisms were generally at the limit of the acceptability thresholds of the reference standards applied in the cases of this study. This indicates the undeniable role that environmental conditions play in the quality of the products produced. This study constitutes a reference in the apprehension of the hygienic and nutritional quality of the Niger soumbala.

Keywords: Soumbala, Physico-Chemistry, Microbiology, Production Environment, Niger.

\section{Introduction}

Les condiments traditionnellement fermentés sont issus d'un savoirfaire empirique très ancien (Azokpota et al., 2011). Il s'agit, selon Koné (2001) et Azokpota et al. (2011), d'un processus de fermentation naturelle des graines protéagineuses et oléagineuses notamment celles de caroubier africain ou néré (Parkia biglobosa), de baobab (Adansonia digitata), d'arachide (Arachis hypogeae), d'oseille de Guinée (Hibiscus Sabdariffa) et de soja (Glycine max). De ce processus résultent très souvent des condiments, notamment le soumbala. Ce condiment possède plusieurs dénominations liées au type de graines oléagineuses transformés, aux pays de provenance et aux ethnies qui le produisent (Odunfa, 1988 ; N'dir, 1997 ; Cheyns et Bricas, 2003 ; Parkouda et al., 2008 ; Parkouda et al., 2009 ; Ibrahim et al., 2011 ; Agbobatinkpo et al., 2013 ; Azokpota et al., 2006 ; Fatoumata et al., 2016). Selon Parkouda et al. (2009), la variabilité des procédés technologiques inter et intra-productrices y joue aussi un rôle important.

Par ailleurs, les résultats des travaux réalisés par N'dir (1994), Benghaly et al. (2005), Parkouda et al. (2009), Ibrahim et al. (2011), Ibeabuchi et al. (2014) et Fatoumata et al. (2016) portant sur les caractéristiques microbiologiques de quelques condiments fermentés ont mis en évidence l'implication des bactéries lactiques, des levures et des moisissures dans ce type de fermentation. Ainsi, de par leurs nombreuses activités lytiques (protéolyse, amylolyse, lipolyse, etc.) (Enujiugha, 2000 et Ibeabuchi et al., 2014), elles modifient les caractéristiques biochimiques, nutritionnelles et sensorielles des graines (protéagineuse et oléagineuses) transformées (Koné, 2001 ). Ces modifications sensorielles participent à la typicité de soumbala qui peut parfois être difficiles à consommer si l'on n'y est pas habitué.

$\mathrm{Au}$ Niger, tout comme dans la plus part des pays ouest-africains, le soumbala est très consommé par la population rurale. Il est utilisé dans la préparation des plats pour rehausser le gout des sauces accompagnant les plats à base de céréales tels que le riz, le mil, le sorgho, le maïs etc. Selon Campaore 
et al. (2013) et Oguntoyinbo (2007), le soumbala constitue également une importante source de protéines pour des familles à faible revenu. Cependant, il existe peu d'études scientifiques portant sur la caractérisation des soumbala produits au Niger. Ainsi, cette présente étude s'inscrit dans ce contexte en vue d'évaluer les caractéristiques physico-chimiques et microbiologiques de deux variantes de soumbala produites au Niger.

\section{Matériel et méthodes}

\subsection{Cadre d'étude}

La collecte des échantillons communautaire de soumbala s'est déroulée dans le département de Torodi (région de Tillabéri), au sein de deux sites. Le premier site est localisé à Pengona et le deuxième site à Makalondi (Figure 1). Aussi, il convient de noter que ces sites (Pengona et Makalondi) ont respectivement servis à l'apprentissage de l'art de production de soumbala d'oseille de guinée et de celui de néré.

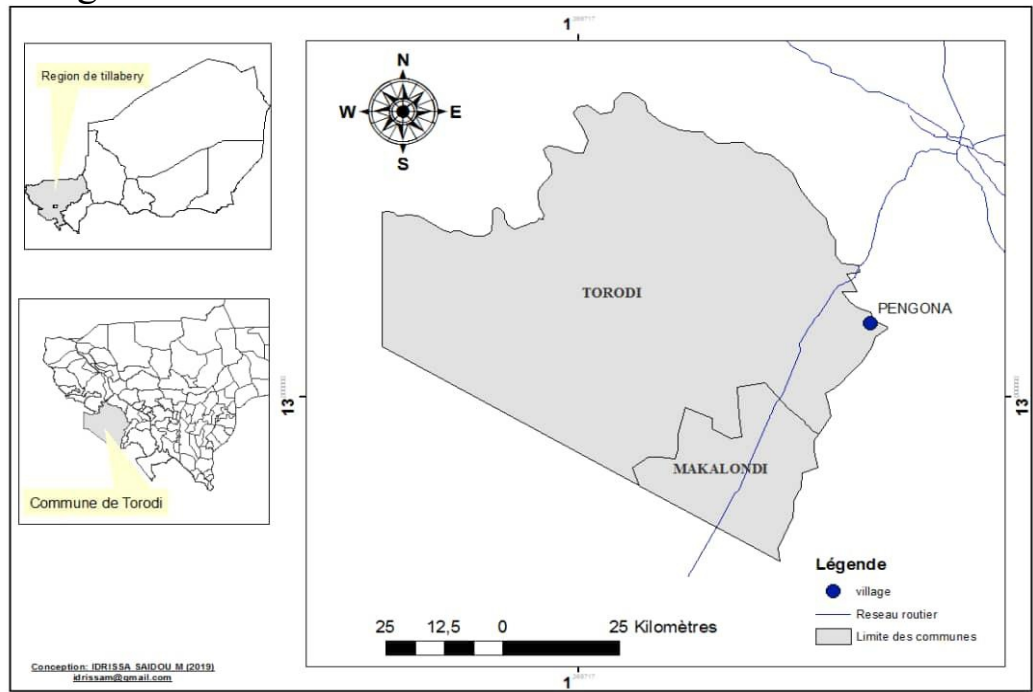

Figure 1. Localisation des sites d'études de la collecte des échantillons de soumbala communautaire

Par ailleurs, les analyses physico-chimiques et microbiologiques de soumbala ont été conduites au sein du Laboratoire de recherches en Hygiènes, Sciences alimentaires et nutritionnelles (LRH-SAN). Ce laboratoire est situé dans la faculté d'Agronomie de l'Université Abdou Moumouni de Niamey.

\subsection{Technique de collecte des échantillons}

Cette étude est réalisée sur deux variétés de soumbala : le soumbala issu de la fermentation des graines de néré (Parkia biglobosa) et celui des graines d'oseille de guinée (Hibiscus sabdariffa). La collecte des échantillons de ces soumbala a été effectuée suivant les milieux de production 
(communautaire et laboratoire). Ainsi, pour chaque variante de soumbala (néré et d'oseille de guinée), deux échantillons ont été respectivement prélevés dans la production communautaire et dans celle du laboratoire : d'où une collecte de quatre (4) échantillons par variante de soumbala. Toutefois, ces échantillons sont représentatifs des deux (2) variantes de soumbala (néré et oseille de guinée) produits dans la zone Ouest du Niger (région de Tillabéry). Aussi, les soumbala ont été produits au laboratoire suivant les diagrammes de production communautaire amélioré. L'amélioration a consisté à la mise en place d'une démarche d'analyse des dangers et points critiques pour leur maîtrise (HACCP).

\subsection{Techniques d'analyse physico-chimiques}

La détermination des teneurs en eau, en matières sèches, en protéines, en lipides et en cendres a été réalisée selon la méthode AOAC (Association of Official Analytical Chemists) (1990). En effet, les teneurs en humidité et en matière sèche ont été calculées suivant la différence de poids du substrat avant et après séchage à l'étuve à $105^{\circ} \mathrm{C}$ durant $6 \mathrm{~h}$. Les teneurs en protéines ont été déterminées selon la méthode Kjeldhal (1990). La matière grasse ou le lipide total a été dosée après extraction au soxhlet. La détermination de teneur en minéraux (Calcium, Sodium et Potassium) a été effectuée selon la méthode de référence de comité inter institut d'étude des techniques analytiques. Cette dernière est la méthode de référence pour la détermination des éléments minéraux dans les végétaux (Pinta, 1973). Le dosage de ces éléments minéraux a été effectué par spectrophotomètre d'absorption atomique de flamme, de marque VARIAN spectrAA 50B. La teneur en glucide a été déterminée par la méthode différentielle. Le calcul a été effectué avec les valeurs déterminées des taux de protéines, de lipides, des cendres et de matières sèches selon la formule différentielle suivante :

$\%$ Glucides totaux / MS = $100-[\% \mathrm{C} / \mathrm{MS}+\% \mathrm{P} / \mathrm{MS}+\% \mathrm{~L} / \mathrm{MS}]$

Avec : \% C/MS : taux des cendres par rapport à la matière sèche ; \% P/MS : taux des protéines par rapport à la matière sèche et \% L/MS : taux des lipides par rapport à la matière sèche.

La valeur énergétique a été calculée à l'aide des coefficients spécifiques d'Atwater notamment pour les protéines, les lipides et les glucides (FAO, 2006).

\subsection{Techniques d'analyse microbiologiques}

Les solutions mères des différents échantillons prélevés ont été préparées selon la méthode ISO 6887-V08-010-6 (2013). Le dénombrement des germes a été effectué suivant les directives des méthodes horizontales ISO. Il s'agit de la norme V 08-015 (1991)/ ISO 4832 pour les coliformes totaux et la norme V 08-017 (1996) pour les coliformes thermotolérants, la norme V 
08-051(1992)/ ISO 4833 pour la flore aérobie mésophile totale , la norme V 08-014 (1984)/ ISO 6888 pour les staphylocoques, la norme V 08-022 (1998) / ISO 7954 pour les levures et moisissures, la norme V 08-030 (1998) / ISO 15214 des lactobacillus et la norme V 08-056 (1994) pour les clostridium sulfito-réducteurs (Guiraud, 2003). En outre, les milieux de cultures et les temps d'incubation ont été réajustés suivant les directives des fabricants comme suit : PCA (Plat Count Agar) à $30^{\circ} \mathrm{C}$ durant $72 \mathrm{~h}, \mathrm{VRBL}$ ( Crital Violet au Rouge Neutre) à $37^{\circ} \mathrm{C}$ durant 24 h, EMB (gélose Eosine Bleue Méthylène) à $37^{\circ} \mathrm{C}$ durant $24 \mathrm{~h}$, Chapman à $37^{\circ} \mathrm{C}$ durant $24 \mathrm{~h}$, YGC ou CGA (Gélose extrait de Levures au Chloramphénicol) à 25 et $37^{\circ} \mathrm{C}$ durant 3 à 5 jours, MRS (Man Rogoza et Sharpe) à $37{ }^{\circ} \mathrm{C}$ durant $24 \mathrm{~h}$, et TSN (Tryptone Sulfite Néomycine) à $46^{\circ} \mathrm{C}$ durant $24 \mathrm{~h}$.

\subsection{Traitement des données}

Pour les données physico-chimiques, le logiciel IBM SPSS Statistics (64 Bit) 20. a servi aux calculs des moyennes et écarts types. Le calcul de corrélation entre les variables a été effectué avec le logiciel $\mathrm{R}$ version 3.5.3 ( $\mathrm{R}$ development Core Team, 2019). Afin de juger la qualité des échantillons collectés, les charges microbiennes ont été comparées aux valeurs de références établies par le comité européen de normalisation des denrées alimentaires : lignes directrices et normes pour l'interprétation des résultats analytiques en microbiologie alimentaire (2011).

\section{Résultats}

\section{III.1. Caractéristiques biochimiques des échantillons de soumbala}

Les résultats présentés dans le tableau 1 indiquent les teneurs en macronutriments et en minéraux des différentes variantes de soumbala.

Les teneurs moyennes en eau des soumbala ont été situées entre 4,61 $\pm 0,21$ et $13,61 \pm 0,34 \%$. En effet, les échantillons de soumbala produit au laboratoire ont présenté les plus faibles taux d'humidité quel que soit le type de soumbala soumis à cette présente étude $(4,61 \pm 0,21 \%$ pour oseille de Guinée et 4,67 \pm $0,19 \%$ pour néré). Par ailleurs, les teneurs moyennes en cendres ont varié de $2,09 \pm 0,14$ à $7,85 \pm 0,16 \%$. Les plus fortes valeurs ont appartenu au soumbala d'oseille de guinée quelle que soit la provenance du soumbala (communautaire, laboratoire). En ce qui concerne les teneurs protéiques, lipidiques, glucidiques et énergétiques, les moyennes ont été respectivement situées entre $28,48 \pm 0,38$ et $48,63 \pm 1,3 \% ; 19,62 \pm 0,56$ et $35,35 \pm 0,47 \%$; $15,41 \pm 0,68 \%$ et $33,91 \pm 1,51 \%$ et $440,18 \pm 1,41 \mathrm{kcal}$ et $529,11 \pm 3,4 \mathrm{kcal}$. Les pourcentages les plus bas en protéines $(28,48 \pm 0,38 \%$ pour la production communautaire et $29,17 \pm 0,63 \%$ pour celle du laboratoire) ont été observés au niveau du soumbala d'oseille de guinée. En revanche, ce soumbala d'oseille de guinée a affiché la plus forte teneur lipidique $(35,35 \pm 0,47 \%)$. La plus 
faible teneur en énergie $(440,18 \pm 1,41 \mathrm{kcal})$ a été enregistrée chez le soumbala de néré produit en milieu communautaire, tandis que la plus forte $(529,11 \pm 3,4$ kcal) teneur a été exprimée par le soumala d'oseille de Guinée produit au laboratoire. Quel que soit le milieu de production considéré, le soumbala du néré a exprimé les teneurs protéiques les plus élevées $(48,63 \pm 1,3 \%$ pour la production communautaire et $39,47 \pm 1,34$ pour celle du laboratoire). Par contre, il a détenu les plus faibles pourcentages en lipides $(20,45 \pm 0,68$ pour la production communautaire et 19,62 $\pm 0,56 \%$ pour celle du laboratoire) et en énergie $(440,18 \pm 1,41$ pour la production communautaire et $470,12 \pm 3,90$ Kcal pour celle du laboratoire). Les valeurs moyennes du taux de glucides ont également présenté des variabilités selon le milieu de production et le type de soumbala considéré. Ainsi, le soumbala de néré communautaire présente un taux de glucides moins élevé par rapport au soumbala du néré produit au laboratoire $(15,41 \pm 0,68$ pour le communautaire et $33,91 \pm 1,51 \%$ pour celui du laboratoire).

Quant aux teneurs en minéraux, les valeurs sont comprises entre 0,45 $\pm 0,07$ et $59,12 \pm 1,3 \mathrm{mg} / 100 \mathrm{~g}$ pour le calcium, $160,61 \pm 10$ et $551,32 \pm 35,55$ $\mathrm{mg} / 100 \mathrm{~g}$ pour le Sodium et $2179,74 \pm 35,9$ et $15812,94 \pm 273,7 \mathrm{mg} / 100 \mathrm{~g}$ pour le potassium. Le soumbala d'oseille de guinée, quel que soit son lieu de production, a exprimé les pourcentages en sodium et en potassium les plus élevés. Par ailleurs, quel que soit le lieu de production, le soumbala de néré a présenté les teneurs calciques les plus importantes, tandis que celui d'oseille de Guinée a affiché les teneurs calciques les plus faibles. 
Tableau 1. Composition biochimique des soumbala

\begin{tabular}{|c|c|c|c|c|c|c|c|c|c|c|}
\hline Échantillons & $\begin{array}{c}\begin{array}{c}\text { Humidité } \\
(\mathrm{g} / \mathbf{1 0 0 g})\end{array} \\
\end{array}$ & $\begin{array}{c}\text { Matière sèche } \\
(\mathrm{g} / \mathbf{1 0 0 g})\end{array}$ & $\begin{array}{c}\text { Protéines } \\
(\mathrm{g} / \mathbf{1 0 0 g})\end{array}$ & $\begin{array}{c}\begin{array}{c}\text { Glucides } \\
(\mathrm{g} / \mathbf{1 0 0 g})\end{array} \\
\end{array}$ & $\begin{array}{c}\text { lipides } \\
(\mathrm{g} / \mathbf{1 0 0 g}) \\
\end{array}$ & $\begin{array}{c}\text { Cendre } \\
(\mathrm{g} / 100 \mathrm{~g})\end{array}$ & $\begin{array}{c}\text { Calcium } \\
(\mathrm{mg} / \mathbf{1 0 0 g}\end{array}$ & $\begin{array}{c}\text { Sodium } \\
(\mathrm{mg} / \mathbf{1 0 0 g})\end{array}$ & Potassium (mg/100g) & $\begin{array}{c}\text { Energie } \\
(\text { Kcal/100g) }\end{array}$ \\
\hline S n com & $13,61 \pm 0,34 \mathrm{c}$ & $86,39 \pm 0,34 \mathrm{a}$ & $48,63 \pm 1,3 \mathrm{c}$ & $15,41 \pm 0,68 \mathrm{a}$ & $20,45 \pm 0,68 \mathrm{a}$ & $2,09 \pm 0,14 \mathrm{a}$ & $59,12 \pm 1,3 \mathrm{~b}$ & $160,61 \pm 10 \mathrm{a}$ & $2192,02 \pm 192,47 \mathrm{a}$ & $440,18 \pm 1,41 \mathrm{a}$ \\
\hline S o lab & $4,61 \pm 0,21 \mathrm{a}$ & $95,39 \pm 0,21 \mathrm{c}$ & $29,17 \pm 0,63 \mathrm{a}$ & $23,58 \pm 1,21 \mathrm{~b}$ & $35,35 \pm 0,47 \mathrm{c}$ & $7,29 \pm 0,37 \mathrm{~b}$ & $0,49 \pm 0,06$ a & $551,32 \pm 35,55 \mathrm{~b}$ & $15812,94 \pm 273,7 \mathrm{c}$ & $529,11 \pm 3,4 \mathrm{c}$ \\
\hline S n lab & $4,67 \pm 0,19 \mathrm{a}$ & $95,33 \pm 0,19 \mathrm{c}$ & $39,47 \pm 1,34 \mathrm{~b}$ & $33,91 \pm 1,51 \mathrm{c}$ & $19,62 \pm 0,56 \mathrm{a}$ & $2,55 \pm 0,07 \mathrm{a}$ & $57,67 \pm 0,85 \mathrm{~b}$ & $194,77 \pm 5,73 \mathrm{a}$ & $2179,74 \pm 35,9 \mathrm{a}$ & $470,12 \pm 3,90 \mathrm{~b}$ \\
\hline S 0 com & $8,16 \pm 0,11 b$ & $91,84 \pm 0,11 \mathrm{~b}$ & $28,48 \pm 0,38 \mathrm{a}$ & $27,78 \pm 0,26 \mathrm{~b}$ & $27,74 \pm 0,51 \mathrm{~b}$ & $7,85 \pm 0,16 b$ & $0,45 \pm 0,07 \mathrm{a}$ & $465,49 \pm 23,45 b$ & $14202,53 \pm 330,45 b$ & $474,67 \pm 2,45 \mathrm{~b}$ \\
\hline p-value & $8,13 \mathrm{e}-09 * * *$ & $8,12 \mathrm{e}-09 * * *$ & $1,61 \mathrm{e}-06 * * *$ & $9,78 \mathrm{e}-06 * * *$ & $1,3 \mathrm{e}-07 * * *$ & $8,06 \mathrm{e}-08 * * *$ & $1,08 \mathrm{e}-11 * * *$ & $2,92 \mathrm{e}-06 * * *$ & $12,6 \mathrm{e}-10 * * *$ & $1,87 \mathrm{e}-07 * * *$ \\
\hline
\end{tabular}

Dans chaque colonne, les valeurs moyennes succédées des mêmes indices, ne sont pas statistiquement différentes au seuil 0,05 .

S o com : échantillons de soumbala d'oseille de Guinée prélevés chez les productrices communautaires; S n lab : échantillons de soumbala de néré produit au laboratoire ; S n com : échantillons de soumbala de néré prélevés chez les productrices communautaires ; S o lab : échantillons de soumbala d'oseille de guinée produit au laboratoire. 


\section{III.2. Corrélation suivant les paramètres biochimiques et physico- chimiques de soumbala}

Le tableau 2 présente les différentes corrélations établies entre les paramètres chimiques et physico-chimiques mesurés et les différents échantillons de soumbala.

Pour les échantillons, des corrélations positives et significatives $(\mathrm{r}=0,92$, $\mathrm{p}=0,0000$ et $\mathrm{r}=0,84, \mathrm{p}=0,0000$ ) ont été observées entre le soumbala du néré communautaire, le taux d'humidité et celui de protéines. Le soumbala d'oseille de guinée a aussi présenté des fortes corrélations positives et significatives $(r=0,90, p=0,0000$ et $r=0,86, p=0,0000)$ entre le pourcentage en énergie et en lipide. En revanche, le soumbala de néré issu de la production communautaire a montré une corrélation négative et très significative $(\mathrm{r}=$ $0,92, p=0,0000$ et $\mathrm{r}=-0,82, \mathrm{p}=0,0000$ ) avec le taux de matière sèche et de glucides.

Pour les paramètres physico-chimiques, une corrélation positive et très significative $(\mathrm{r}=0,91, \mathrm{p}=0,0000)$ a été observé entre la teneur protéique et la teneur calciques des échantillons.

La teneur en lipides a aussi présenté des corrélations positives et très significatives $(r=0,85, p=0,0000 ; r=0,95, p=0,0000 ; r=0,93, p=0,0000$ et $r$ $=0,89, \mathrm{p}=0,0000$ ) avec les taux de cendre, de sodium, de potassium et d'énergie. Des fortes corrélations positives et significatives $(r=0,94$, $\mathrm{p}=0,0000$ et $\mathrm{r}=0,98, \mathrm{p}=0,000$ ) ont été observées entre le taux de cendre et les teneurs en sodium et potassium des différents produits. Par contre, des corrélations négatives et très significatives ont été notées entre la teneur protéique et les taux de cendre $(\mathrm{r}=-0,92, \mathrm{p}=0,0000)$, de sodium $(\mathrm{r}=-0,91$, $\mathrm{p}=0,0000)$ et de potassium $(\mathrm{r}=-0,90, \mathrm{p}=0,0000)$. Le taux de lipides a également présenté une corrélation négative et très significative $(r=-0,89$, $\mathrm{p}=0,0000$ ) avec le pourcentage calcique. Aussi, ce dernier a été négativement corrélée avec les taux de sodium et de potassium $(r=-0,96, p=0,0000$ et $r=-$ $0,99, \mathrm{p}=0,0000)$. 
Tableau 2. Corrélation entre les différentes variables biochimiques et physico-chimiques

\begin{tabular}{|c|c|c|c|c|c|c|c|c|c|c|c|c|c|c|}
\hline \multirow[t]{2}{*}{ Variables } & \multicolumn{4}{|c|}{ Echantillons } & \multirow[t]{2}{*}{ Humidité } & \multirow{2}{*}{$\begin{array}{l}\text { Matière } \\
\text { sèche }\end{array}$} & \multirow[t]{2}{*}{ Protéines } & \multirow[t]{2}{*}{ Glucides } & \multirow[t]{2}{*}{ lipides } & \multirow[t]{2}{*}{ Cendre } & \multirow[t]{2}{*}{ Calcium } & \multirow[t]{2}{*}{ Sodium } & \multirow[t]{2}{*}{ Potassium } & \multirow[t]{2}{*}{ Energie } \\
\hline & S 0 com & S n lab & S n com & S o lab & & & & & & & & & & \\
\hline Humidité & 0,06 & $-0,48$ & $0,91 * *$ & $-0,49$ & 1 & & & & & & & & & \\
\hline $\begin{array}{l}\text { Matière } \\
\text { sèche }\end{array}$ & $-0,06$ & 0,48 & $-0,91 * *$ & 0,49 & $-0,99 * *$ & 1 & & & & & & & & \\
\hline Protéines & $-0,54$ & 0,20 & $\mathbf{0 , 8 3} * *$ & $-0,49$ & 0,65 & $-0,65$ & 1 & & & & & & & \\
\hline Glucides & 0,21 & $\mathbf{0 , 7 3} * *$ & $-0,81 * *$ & $-0,13$ & $-0,77 * *$ & $0,77 * *$ & $-0,46$ & 1 & & & & & & \\
\hline lipides & 0,17 & $-0,55$ & $-0,48$ & $\mathbf{0 , 8 6 * *}$ & $-0,43$ & 0,43 & $-0,78 * *$ & $-0,7$ & 1 & & & & & \\
\hline Cendre & 0,63 & $-0,52$ & $-0,62$ & 0,51 & $-0,39$ & 0,39 & $-0,91 * *$ & 0,13 & $\mathbf{0 , 8 5 * *}$ & 1 & & & & \\
\hline Calcium & $-0,57$ & 0,56 & 0,59 & $-0,57$ & 0,39 & $-0,39$ & $0,90 * *$ & $-0,08$ & $-0,89 * *$ & $-0,98 * *$ & 1 & & & \\
\hline Sodium & 0,41 & $-0,49$ & $-0,61$ & $\mathbf{0 , 7 0 * *}$ & $-0,47$ & 0,47 & $-0,90 * *$ & 0,09 & $0,95 * *$ & $0,94 * *$ & $-0,96 * *$ & 1 & & \\
\hline Potassium & 0,50 & $-0,57$ & $-0,57$ & 0,64 & $-0,39$ & 0,39 & $-0,90 * *$ & 0,05 & $0,93 * *$ & $0,98 * *$ & $-0,99 * *$ & $0,97 * *$ & 1 & \\
\hline Energie & $-0,06$ & $-0,15$ & $-0,68$ & $\mathbf{0 , 9 0 * *}$ & $-0,75^{* *}$ & $0,75^{* *}$ & $-0,76$ & 0,22 & $0,89 * *$ & 0,68 & $-0,72$ & $\mathbf{0 , 8 3} * *$ & 0,77 & 1 \\
\hline
\end{tabular}

* : Corrélation significative à $\mathrm{p}<0,05 . * *:$ Corrélation significative à $\mathrm{p}<0,01$. Les coefficients de corrélation indiquant une relation de forte interaction $(\mathrm{p}<0,01)$ ont été considérés.

S o com : échantillons de soumbala d'oseille de Guinée prélevés chez les productrices communautaires; $\mathbf{S} \mathbf{n}$ lab : échantillons de soumbala de néré produit au laboratoire ; $\mathbf{S} \mathbf{n} \mathbf{~ c o m ~ : ~ e ́ c h a n t i l l o n s ~ d e ~ s o u m b a l a ~ d e ~ n e ́ r e ́ ~ p r e ́ l e v e ́ s ~ c h e z ~ l e s ~ p r o d u c t r i c e s ~ c o m m u n a u t a i r e s ~ ; ~} \mathbf{S}$ o lab : échantillons de soumbala d'oseille de guinée produit au laboratoire. 


\section{III.3. Profil microbien des échantillons de soumbala}

Les résultats présentés dans la figure 3 indiquent une charge acceptable en coliformes totaux $(2,67 \pm 0,88$ à 46,23 $\pm 4,07)$ pour tous les échantillons de soumbala analysés. Toutefois, les échantillons de soumbala de néré prélevés en milieu communautaires ont affiché un taux élevé en coliformes totaux $(55,3 \pm 2,7)$. Il en a été de même pour les coliformes fécaux (Thermotolérants) où la teneur $(27,33 \pm 1,76)$ a été au-dessus de la limite d'acceptabilité (Figure 4).

En outre, tous les échantillons analysés ont présenté de différences significatives au niveau des profils microbiologiques des coliformes totaux $(\mathrm{p}=0,000022)$, des coliformes fécaux $(\mathrm{p}=0,0000052)$, des staphylocoques $(\mathrm{p}=0,0043)$, des Lactobacillus $(\mathrm{p}=0,021)$, des moisissures $(\mathrm{p}=0,025)$, des Clostridum sulfito réducteurs $(\mathrm{p}=0,0000057)$. Par contre, la fréquence $\mathrm{d}$ 'isolement de la flore aérobie mésophile totale (Figure 2), des levures (Figure 6), n'a pas montré de différence statistique $(\mathrm{p}=0,19)$.

Par ailleurs, une absence de souches de Clostridium a été observée au niveau de soumbala de néré provenant des deux milieux de production (Figure $8)$. En ce qui concerne les lactobacillus, les résultats inscrits dans la figure 5 indiquent l'existence d'une flore très abondante avec une différence légèrement significative entre les échantillons $(\mathrm{p}=0,021)$. 

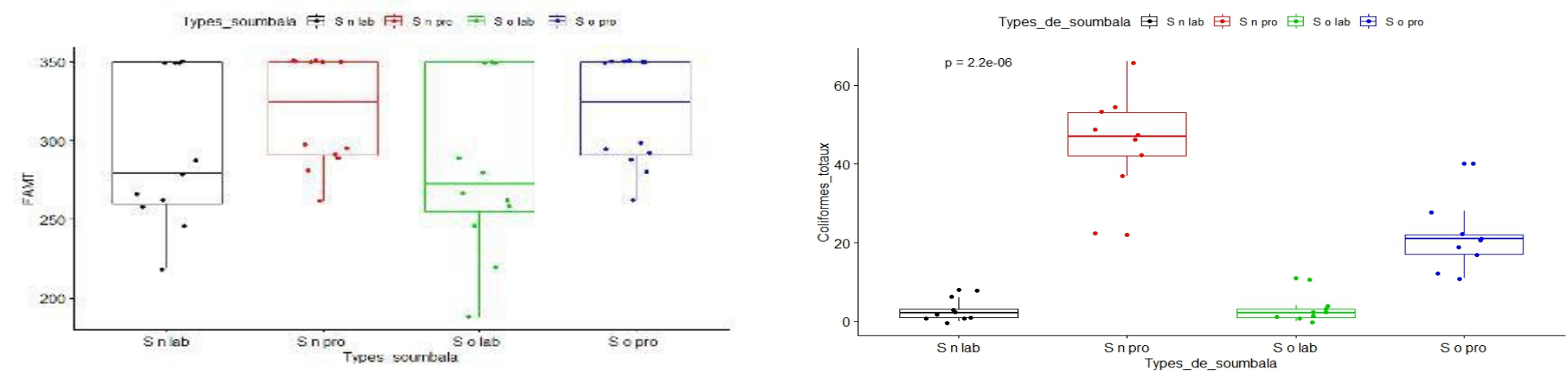

Figure 2. Fréquence d'isolements de la flore aérobie mésophile totale

Figure 3. Fréquence d'isolements des coliformes totaux
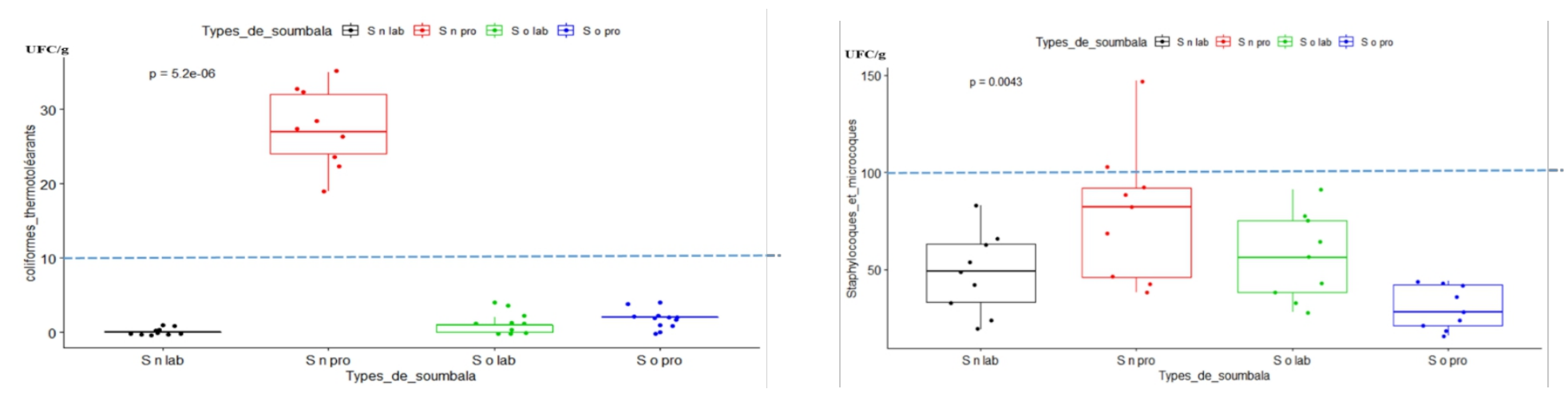

Figure 4. Fréquence d'isolements des thermotolérants microcoques

Figure 5. Fréquence d'isolements des staphylocoques et 


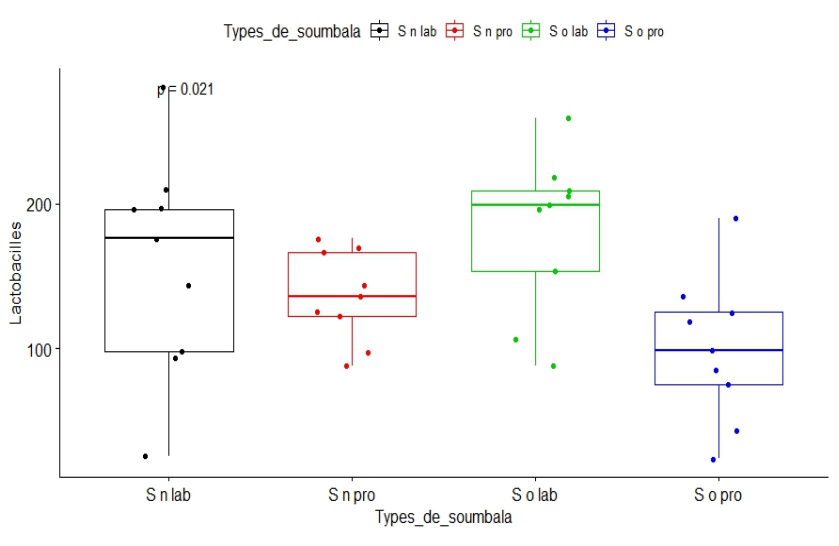

Figure 6. Fréquence d'isolements des Lactobacillus

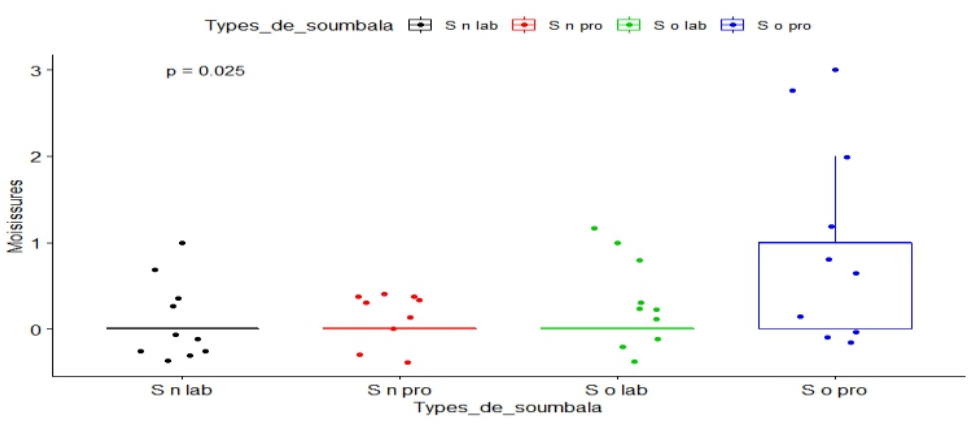

Figure 8. Fréquence d'isolements des moisissures réducteurs

Les boites à moustaches donnent les quartiles $25 \%, 50 \%, 75 \%$ et $100 \%$ des distributions. La longueur de la boîte indique : les intervalles interquartiles. Les points: les valeurs aberrantes. Les lignes horizontales épaisses en trait plein: les valeurs des médianes. Les moustaches: les interquartiles fois 1,5 .

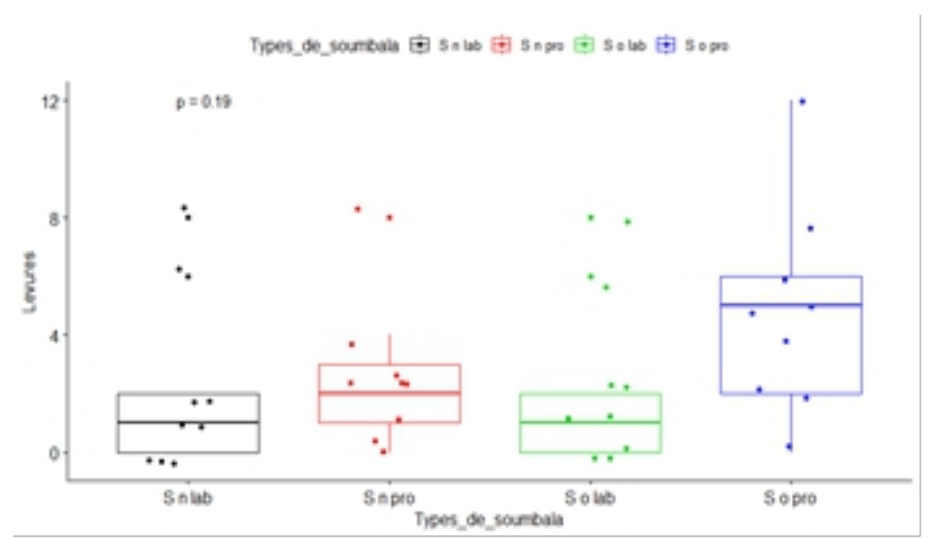

Figure 7. Fréquence d'isolements des levures

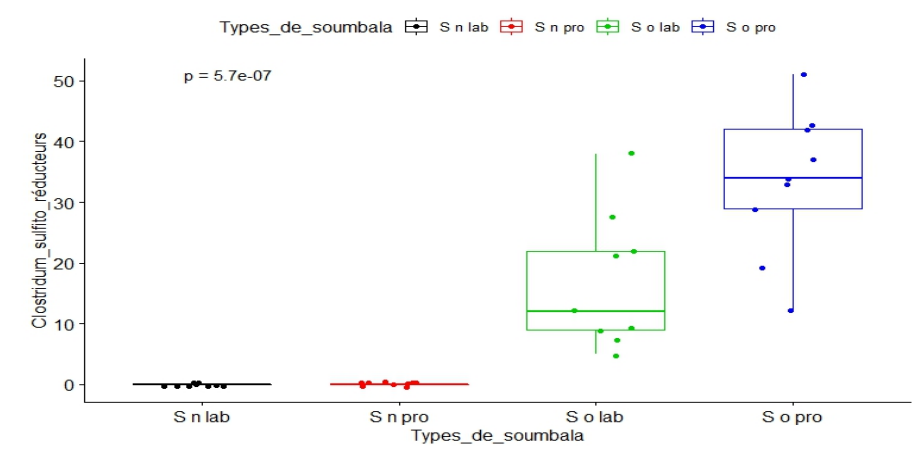

Figure 9. Fréquence d'isolements des Clostridiums sulfito- 


\section{Discussion}

L'analyse des résultats montre que le Soumbala de néré provenant de la production communautaire possède à la fois la plus forte teneur moyenne en eau et le plus grand pourcentage protéique. En outre, il présente des teneurs en lipides, en énergie et en cendres les moins élevées. Ainsi la faible teneur lipidique déterminée dans cet échantillon pourrait être expliquée par la possibilité de survenue des réactions d'oxydation provoquée par la forte teneur en eau du produit. Aussi, le fort pourcentage protéique pourrait être relatif aux caractéristiques variétales (Fatoumata et al., 2016) et/ou aux types de microorganismes ayant proliféré lors du processus de fermentation de ce produit. Selon Ouoba et al. (2003), la nature de l'espèce et la souche de bactéries qui se développent influent fortement sur la teneur protéique du produit. C'est ainsi que les essais de production réalisés par ajout de ferments ont montré que les souches de Bacillus subtilis présentent de forte activité protéolytique par rapport aux Bacillus pumilus (Ouoba et al., 2003). En outre, il se pourrait également que la forte teneur en eau de ce produit favoriserait la croissance de ces types de germes, après les $48 \mathrm{~h}$ suivant l'arrêt des procédés de fermentation : d'où l'existence des corrélations positives et significatives observées entre les taux d'humidité, de protéines et le soumbala du néré communautaires. Il convient de noter que la teneur moyenne en eau observée dans le soumbala de néré prélevé en milieu communautaire est quasi similaire à celles rapportées par Appiah et al. (2012) dans les échantillons du soumbala de néré produit au Ghana. Ce qui n'a pas été le cas dans les échantillons du soumbala de néré produit au Sénégal (N'dir et al., 1997) et au Nigeria (Gernah et al., 2007) où les teneurs ont été plus élevées. Le pourcentage de protéines est également dans le même ordre de grandeur que celui rapportés par Appiah et al-(2012) sur le soumbala de néré produit au Ghana.

Par ailleurs, le soumbala d'oseille de Guinée produit au laboratoire enregistre les plus fortes teneurs moyennes en lipides, en énergie, en sodium et en potassium. Cette particularité de compositions biochimique et physicochimique de cet échantillon pourrait être allouée aux effets bénéfiques du procédé de séchage mis en place. En effet, le séchage inhibe le processus de lipolyse de la matière grasse contenue dans les aliments (Nout et al., 2003). Aussi la teneur en lipides de certaines variétés de graines non fermentées d'oseille de Guinée est souvent élevée (Diouf, 2013). La forte valeur énergétique pourrait être indirectement liée au pourcentage lipidique du produit. En effet, le coefficient de conversion du pourcentage de nutriments en valeur énergétique alloue $9 \mathrm{Kcal}$ pour $1 \mathrm{~g}$ de lipides (FAO, 2006). Ce rapport de concordance favoriserait donc les produits présentant une forte teneur en matière grasse par rapport à ceux moins riches en matière grasse. Les résultats de corrélations entre les différentes variables viennent renchérir cette hypothèse car il existe de fortes corrélation positives entre ces différentes 
variables. Les différences observées, notamment aux niveaux des taux de cendres et des minéraux, s'expliqueraient par la diversité des procédés (Fatoumata et al., 2016). De même, certaines productrices communautaires s'adonnent à l'ajout des additifs ou à la combinaison des matières premières (graines transformées) afin d'améliorer le goût ou la texture du produit fini (Cheyns et Bricas, 2003). En outre, plus le produit est riche en cendre, plus sa teneur en différents minéraux est élevée : d'où la présence des fortes corrélations positives et significatives entre le taux de cendre et la teneur en sodium et potassium des différents produits.

Par ailleurs, l'analyse du profil microbien des différents échantillons de soumbala met en évidence la présence d'une population microbienne très diverse. Ces microorganismes sont aussi présents à des proportions variables selon le type de produit et le milieu de provenance. Ceci a été mis en évidence par l'existence de différence significative au niveau de la fréquence d'isolement des coliformes totaux et fécaux, des staphylocoques, des lactobacillus, des Moisissures (Aspergillus) et des Clostridum sulfito réducteurs dans les différents échantillons. Ainsi, le soumbala de néré prélevé en milieu communautaire présente la teneur la plus élevée en coliformes fécaux. Cette différence pourrait être liée aux conditions environnementales et techniques de mise en œuvre de certaines opérations unitaires. En effet, les productrices communautaires sont confrontées à des difficultés d'accès à l'eau potable. Cette dernière est vendue à des coûts très élevés selon les périodes de l'année. Pourtant, le procédé de rinçage de graines décortiquées exige une quantité importante d'eau. Afin de pallier aux coûts élevés d'eau, certaines productrices communautaires utilisent l'eau des marres et marigots en saisons hivernales. Cependant, cette eau est également utilisée pour le pâturage des animaux. Aussi, après le procédé de fermentation, l'opération de confections de galettes du produit s'effectue à la main dans un environnement très favorable au développement de ces types de germes. En effet, les concessions des productrices de soumbala (communautaire) abritent des animaux domestiques (chèvres, moutons, etc.) laissés en pâturage dans l'espace réservé à la production.

En outre, la présence élevée des staphylocoques surtout dans les échantillons de soumbala d'oseille de guinée, bien que cette présence n'excède pas la limite de tolérance, pourrait s'expliquer par l'implication de ces germes dans le processus de fermentation. Ainsi, en absence de la souche de Staphylococcus, selon Benghaly et al. (2005), le produit obtenu n'a ni le goût, ni l'odeur caractéristique du bi-kalga (condiment traditionnel du Burkina issu de la fermentation des graines d'oseille de guinée). Cet auteur indique également que la souche Staphylococcus spp. est probablement responsable de la genèse des composés d'arômes qui intervient au cours de la maturation. 
Aussi, l'absence des souches de Clostridium sulfito-réducteurs au niveau des échantillons de soumbala de néré pourrait être liée aux techniques locales de mise en fermentation de ce condiment. En effet, le dispositif de fermentation des graines de néré utilisé par les productrices communautaires du Niger présente quelques particularités. Il laisse une entrée minime d'air. Ceci pourrait favoriser la sélection d'un certains types de germes aux détriments des autres puisque, selon Guiraud (2003), les Clostridium sont des germes qui se développent dans les conditions d'anaérobies strictes. Pour N'dir et al. (1997) et Fatoumata et al. (2016), la présence des germes comme les streptocoques D, les Clostridium sulfito réducteurs et certains staphylocoques résultent des mauvaises pratiques d'hygiènes. C'est ce qui explique les cas d'altération de soumbala dans lesquels ils s'y trouvent.

Par ailleurs, la présence très importante des souches du genre Lactobacillus dans les différents échantillons ne constitue pas un handicap sur la qualité du soumbala. En effet, des travaux antérieurs (Ouoba et al., 2007 ; Mohamadou et al., 2007 ; Compaoré et al., 2013 ; Taale et al., 2013 et 2015) ont démontré les effets bénéfiques de ces souches sur la préservation de la qualité hygiénique de ces types de condiments malgré la présence des germes présumés pathogènes ( $E$. coli, Staphylocoques, certaines moisissures, etc.). Pour Ouoba et al. (2007), ces souches créent une symbiose qui rend le milieu hostile au développement des germes d'altération ou pathogènes.

Toutefois, la conformité de certains résultats observée relativement aux fréquences d'isolements des thermotolérants, des levures, des moisissures, des staphylocoques et des microcoques pourrait s'expliquer par des séances de sensibilisation sur les règles d'hygiènes réalisées au cours d'un stage d'apprentissage des procédés de production du soumbala. Ces séances ont été animées en amont de la collecte des échantillons. Quant aux échantillons de soumbala produit au laboratoire, leurs profils microbiens se démarquent nettement de soumbala communautaire. Ceci s'expliquerait par la mise en place de la démarche HACCP lors de la conception de ces produits. Cette démarche aurait ainsi diminué le risque de contamination par les germes présents dans l'environnement. Globalement, ces résultats corroborent ceux des travaux réalisés par plusieurs auteurs (Odunfa, 1985 ; Antai et Ibrahim, 1986 ; N'dir et al., 1997; Ouoba et al., 2003 ; Azokpota et al., 2006 , 2007 ; 2011 ; Ibrahim et al., 2011 ; Agbotinkpo et al., 2013 ; Fatoumata et al., 2016) qui stipulent que les microorganismes contenus dans la moutarde africaine de néré et d'oseille de guinée sont nombreux et appartiennent à différentes catégories de bactéries et de champignons.

\section{Conclusion}

Il ressort de ce travail que des échantillons de soumbala étudiés présentent un intérêt nutritionnel eu égard à leur composition chimique. Aussi, 
cette composition est liée à la typologie de soumbala et le milieu dans lequel le soumbala est produit. Par ailleurs, l'évaluation de la qualité hygiénique de ces échantillons a permis d'élaborer le profil microbien de soumbala de néré et de celui d'oseille de Guinée. Ainsi, il existe une différence très significative dans la fréquence d'isolements de la flore aérobie mésophile totale, des coliformes, des levures et moisissures, des staphylocoques et microcoques, des Clostridium sulfito-réducteurs et Lactobacillus dans les différents échantillons suivant leur milieu de provenance. Toutefois, les teneurs de ces microorganismes sont en général à la limite des seuils d'acceptabilité des normes de références appliquées dans les cas de cette étude. Au vu de ces résultats, le maintien des conditions de production exempte de contamination et respectant l'art de l'activité sont des éléments clés pour l'élaboration de soumbala de bonne qualité nutritionnelle et hygiénique. Enfin, la caractérisation phylogénétique et fonctionnelle de la microflore des Lactobacillus dénombrée apportera d'amples informations sur le profil microbien de ces condiments en général.

\section{References :}

1. Agbobatinkpo, P.B., Thorsen, L., Nielsen, D.S., Azokpota P., Akissoe, N., Hounhouigan, D.J. and Jakobsen, M. (2013). Biodiversity of aerobic endospore-forming bacterial species occurring in Yanyanku and Ikpiru, fermented seeds of Hibiscus sabdariffa used to produce food condiments in Benin. International Journal of Food Microbiology, 163: 231-238.

2. AOAC (1990). Official Methods of Analysis. Association of Official Analytical Chemists, $15^{\text {th }}$. Edition, Washington D.C.

3. Appiah,F.,Oduro,I.Ellis,W.O., and Adu, G. (2012). Comparative assessment of the performance of Parkia biglobosa, Glycine max and Treculia africana in the production of a local condiment in Ghana. African Journal of Food Science,6(5): 111-116

4. Azokpota, P., Hounhouigan, D.J. and Nago, M.C. (2006). Microbiological and chemical changes during the fermentation of African locust bean Parkia biglobosa, to produce afitin, iru and sonru, three traditional condiments produced in Benin, International Journal of Food Microbiology, 107: 304-309.

5. Azokpota, P., Houndenoukon, M.S.E., Hounhouigan, D.J., Nago, M.C. and Jakobsen, M. (2011). Evaluation of yanyanku processing, an additive used as starter culture to produce fermented food condiments in Benin. Journal of Food Processing and Preservation, 35: 813-821.

6. Bengaly, M.D., Augustin, B.B. and Alfred, S.T. (2005). Les graines de Hibiscus sabdariffa $\mathrm{L}$ fermentées : un condiment ouest africain riche en protéines ; potentiel véhicule pour la fortification en fer. Article 
présenté lors de la Conférence sur: Maîtrise des procédés en vue d'améliorer la qualité et la sécurité des aliments. Utilisation des OGM. Analyse des risques en agroalimentaire Ouagadougou, 8-11 Novembre 2005.

7. Cheyns E. and Bricas, N. (2003). La construction de la qualité des produits alimentaires : le cas du soumbala, des céréales et des viandes sur le marché de Ouagadougou au Burkina Faso = Food product quality development processes. Case studies on soumbala, cereal and meat products on the Ouagadougou market. Montpellier : CIRAD, 82 p. (Série ALISA).

8. Compaoré, C.S., Nielsen, D.S., Ouoba, L.I.I., Berner, T.S., Nielsen, K.F., Sawadogo-Lingani, H, Diawara, B, Georges A.O, Jakobsen,M and Thorsen, L. (2013). Co-production of surfactin and a novel bacteriocin by Bacillus subtilis subsp. Subtilis $\mathrm{H} 4$ isolated from Bikalga, an African alkaline Hibiscus sabdariffa seeds fermented condiment, International Journal of Food Microbiology (2013), doi: 10.1016/j.ijfoodmicro.2013.01.013.

9. Comité Européen de normalisation des denrées alimentaires: lignes directrices et normes pour l'interprétation des résultats analytiques en microbiologie alimentaire (2011). Ce document est disponible surinternetàl'adresse:http://www.mapaq.gouv.qc.ca/fr/Publications/re cueil.pdf.

10. Diouf, A.B.K. (2013). Performances Zoo technico-Economiques permises par l'incorporation de la farine de graines de bissap (Hibiscus Sabdariffa) de la variété rouge dans l'alimentation des poulets de chair au Sénégal. THESE soutenue en vue d'obtention du grade de Docteur en médecine vétérinaire. Université Cheikh Anta Diop de Dakar, 121 p.

11. Enujiugha, V.N. and Agbede, J.O. (2000). Nutritional and antinutritional charactéristics of African oil bean Pentaclethra macrophylla Benth, seeds. Applied Tropical Agriculture, 5: 11-14.

12. FAO. (2006).Corporate Document Repository.Calculation of the Energy Content of Foods-Energy Conversion Factors. [http:/www.fao.org/ag]. Consulté le 20/10/2019.

13. Fatoumata, C., Soronikpoho, S., Souleymane T., Kouakou, B. and Marcellin, J.K. (2016). Caractéristiques biochimiques et microbiologiques de moutardes africaines produites à base de graines fermentées de Parkia biglobosa et de Glycine max, vendues en Côte d'Ivoire. International Jounal of Biological and Chemical Sciences. 10(2): 506-518.

14. Pinta, M. (1973). Méthodes de référence pour la détermination de la teneur des éléments minéraux dans les végétaux : détermination des 
éléments $\mathrm{Ca}, \mathrm{Mg}, \mathrm{Fe}, \mathrm{Zn}$ et $\mathrm{Cu}$ par absorption atomique. In : Contrôle de l'alimentation des plantes cultivées, Oléagineux, 28 (2) : 87-92. Le contrôle de l'alimentation des plantes cultivées : Colloque Européen et Méditerranéen, 3., Budapest (Hu), 1972/09/04-08.ISSN 0030-2082.

15. Gernah, D.I., Inyang, C.U. and Ezeora, N.L. (2007). Incubation and fermentation of African locust beans. Parkia biglobosa, in production of "dawadawa", Journal of Food Process \& Preserv, 31: 227-239.

16. Guiraud, J.P. (2003). Microbiologie alimentaire. Dunod, Paris, 2003, pour la nouvelle présentation. ISBN 2100072595. $651 \mathrm{p}$.

17. Ibrahim, A.D., Sani, A. and Shinkafi, A.S. (2011). Production, microbial and physico-chemical evaluation of 'dawadawan botso' (a condiment) produced by the fermentation of Hibiscus sabdariffa seeds. International Jounal of Biological and Chemical Sciences. 5(6): 2481 2490 .

18. Ibeabuchi, J.C., Olawuni, I.A., Iheagwara, M.C., Ojukwu, M. And Ofoedu, C. E. (2014). Microbiological Evaluation of 'Iru' and 'OgiriIsi' Used As Food Condiments. Journal of Environmental Science, Toxicology and Food Technology, 8: 45-50.

19. Koné, S. (2001): Fabrication du 'sumbala', gate Information Service (gtz, PO Box 5180, 65726 Eschborn, Germany), Food processing field, Disponible file: F030f.pdf / doc. 8 p.

20. Mohamadou, B.A., Mbofung, C.M.F. and Thouvenot, D. (2007). Functional Potentials of a product from Traditional Biotechnology: Antioxidant and Probiotic Potential of Mbuja produced by fermentation of Hibiscus sabdariffa seeds in Cameroon. Journal of Food Science and Technology. 5(2): 164-168.

21. Ndir, B., Hbid C.., Cornelius C., Roblain, D., Jacques P.H., Vanhentenry, C.k.F., Diop, M. and Thonart, P. (1994). Propriétés antifongiques de la microflore sporulée du nététu. Cahiers. Agricultures. 3: 23-30.

22. Ndir, B., Gningue, R.D, Keita, N.G, Souane, M., Laurent, L., Cornelius, C. and Thonart, P. (1997). Microbiological and organoleptic characteristics of commercial netetu, Cahiers d'étude et de recherche francophones / Agricultures 6: 299-304.

23. NF EN ISO 6887-V08-010-6. (2013). Microbiologie des alimentsPréparation des échantillons, de la suspension mère et des dilutions décimales en vue de l'examen microbiologique-Partie 6: règles spécifiques pour la préparation des échantillons prélevés au stade de production primaire, $5 \mathrm{p}$.

24. Nout, R., Hounhouigan, J.D., et Van Boekel, T. (2003). Les aliments : Transformation, Conservation et Qualité. CTA, Postbus 380,6700 AJ Wageningen, Pays Bas. ISBN 90-5782-124-9. 
25. Odunfa, S.A. (1985). Biochemical changes in fermenting African Locust Bean Parkia biglobosa, during. iru Fermentation. Journal of Food Science and Technology, 20: 295-303.

26. Ouoba, L.I.I., Cantor, M.D., Diawara, B., Traore, A.S. and Jakobsen, M. (2003). Degradation of African Locust Bean oil by Bacillus subtilis and Bacillus pumilus isolated from soumbala, a fermented African Locust Bean condiment, Journal of Applied Microbiology, 95: 868873.

27. Ouoba, L.I.I., Diawara, B., Jespersen, L. and Jakobsen, M. (2007). Antimicrobial activity of Bacillus subtilis and Bacillus pumilus during the fermentation of African locust bean Parkia biglobosa, for soumbala production. Journal of Applied Microbiology, 102: 963-970.

28. Oguntoyinbo, F.A., Sanni, A.I., Franz, C.M.A.P. and Holzapfel, W.H. (2007). In vitro fermentation studies for selection and evaluation of Bacillus strains as starter cultures for the production of okpehe, a traditional African fermented condiment. International Jounal of food Microbiology, 113: 208-218.

29. Parkouda, C., Diawara, B. and Ouoba, L. I.I. (2008). Technology and physico-chemical characteristics of bikalga, alkaline fermented seeds of Hibiscus sabdariffa, African Journal of Biotechnology, 7: 916-922.

30. Parkouda, C, Nielsen, D.S, Azokpota, P., Ouoba, L.I.I, Amoa-Awua, W.K., Thorsen, L., Hounhouigan, D.J., Jensen, J.S., Tano-Debrah, K., Diawara, B. and Jakobsen, M. (2009). The microbiology of alkalinefermentation of indigenous seeds used as food condiments in Africa and Asia. Critical Reviews in Microbiology, 35(2): 139-156.

31. Taale E., Savadogo A., Zongo C., Ilboudo A.J. and Traore A.S. (2013). Bioactive molecules from bacteria strains: case of bacteriocins producing bacteria isolated from foods. Current Research in Microbiology and Biotechnology, 1(3): 80-88.

32. 32.Taale E., Savadogo A., Zongo C., Somda M.K., Sereme S.S., Karou S.D., Soulama I. and Traore A.S. (2015). Characterization of Bacillus species producing Bacteriocin-like inhibitory substances (BLIS) isolated from fermented food in Burkina Faso. International Journal of Advanced Research in Biological Sciences, 2(4): 279-290. 